\title{
A database for medicinal plants used in the treatment of diabetes and its secondary complications
}

\author{
Nirmala Arulrayan, Saradha Rangasamy, Eliza James, Daisy Pitchai* \\ Bioinformatics Centre, Dept.of Biotechnology and Bioinformatics, Holy Cross College, Tepakulam, Tiruchirapalli-620002; \\ Daisy Pitchai*- Email: daisylesslie@yahoo.com; * Corresponding author \\ received May 28, 2007; revised June 07, 2007; accepted June 13, 2007; published online June 26, 2007
}

\begin{abstract}
:
Effective treatment of diabetes is increasingly dependent on active constituents of medicinal plants capable of controlling hyperglycemia as well as its secondary complications. Sensing the importance of documenting such medicinal plants, here we describe a web database containing information (name, literature citation, active compounds and few related full text articles) of the diabetes medicinal plants exhibiting hypoglycemic, antioxidant and antimicrobial effects.
\end{abstract}

Availability: http://www.autogeneralfilters.com/holycross/Home.html

Keywords: diabetes; medicinal plants; database; literature; active principles; antioxidant; antimicrobial properties

\section{Background:}

Diabetes mellitus is possibly the world's largest growing metabolic disorder and around 30 million people around the world suffer from this disorder.[1] Diabetes can be defined as a group of syndromes characterized by hyperglycemia, altered metabolism of lipids, carbohydrates and proteins.[2] The complications of diabetes include vascular diseases, eye disorders, renal disorders and a host of secondary infections. [3] The treatment of diabetes with synthetic drugs is generally not preferred because of its high cost and the range of side effects caused. Hence development of traditional or alternative medicine is needed. Herbal drugs constitute an important part of traditional medicine and literature shows that there are more than 400 plant species showing antidiabetic activity. $[4,5]$ Our interest is to study these anti diabetic medicinal plants. Research findings suggest that many of these plants control diabetes by exhibiting hypoglycemic and antioxidant effects. The secondary infections associated with diabetes are also restricted to antimicrobial effect by plant products. Sensing the opportunities provided and in an effort to translate research into technology, this database 'DIAB' was created to document the research literature available on these medicinal plants and their active compounds.

\section{Methodology:}

\section{Step 1: Data collection}

Data of antidiabetic plants were collected from various literature sources such as PubMed [6], ScienceDirect [7], Biomed Central [8] Springerlink [9], Scirus [10], Wiley journals [11] and also from folklore medicinal usage. The current dataset contains information for about 258 genus and 287 species of plants exhibiting antidiabetic effect. Of these 287 antidiabetic plants, 129 species show proven antioxidant effect and 53 species exhibit antimicrobial effect besides their hypoglycemic activity.

\section{Step 2: Database Design}

The database is developed using MS Access as back-end and ASP.NET as front-end on a Windows platform and updated regularly.

\section{Step 3: Features of DIAB}

Three separate links (antidiabetic, antioxidant, and antimicrobial) are provided to access the literature citations of antidiabetic plants showing hypoglycemic, antioxidant and antimicrobial properties respectively. The record entry consists of the following literature: plant name, part investigated, authors and abstract. The 'Active principles' link provides information (plant name, part investigated, active compounds and properties) on the 46 compounds isolated from the antidiabetic medicinal plants. The 'Articles' link provides few free text articles for further reading. A screen shot of the database is given in figure 1.

\section{Utility:}

In the current scenario there are several other databases which give information on medicinal plant name, distribution, drugs formulated and usage in treatment of diabetes. We believe that the freely available database called DIAB will give supplementary information on the antidiabetic plants capable of controlling some secondary complications. This database finds utility to the scientific community for a quick review on plants for diabetes medicinal plant research and provides enormous scope for development of drugs. 


\section{www.bioinformation.net}

\section{Web Database}

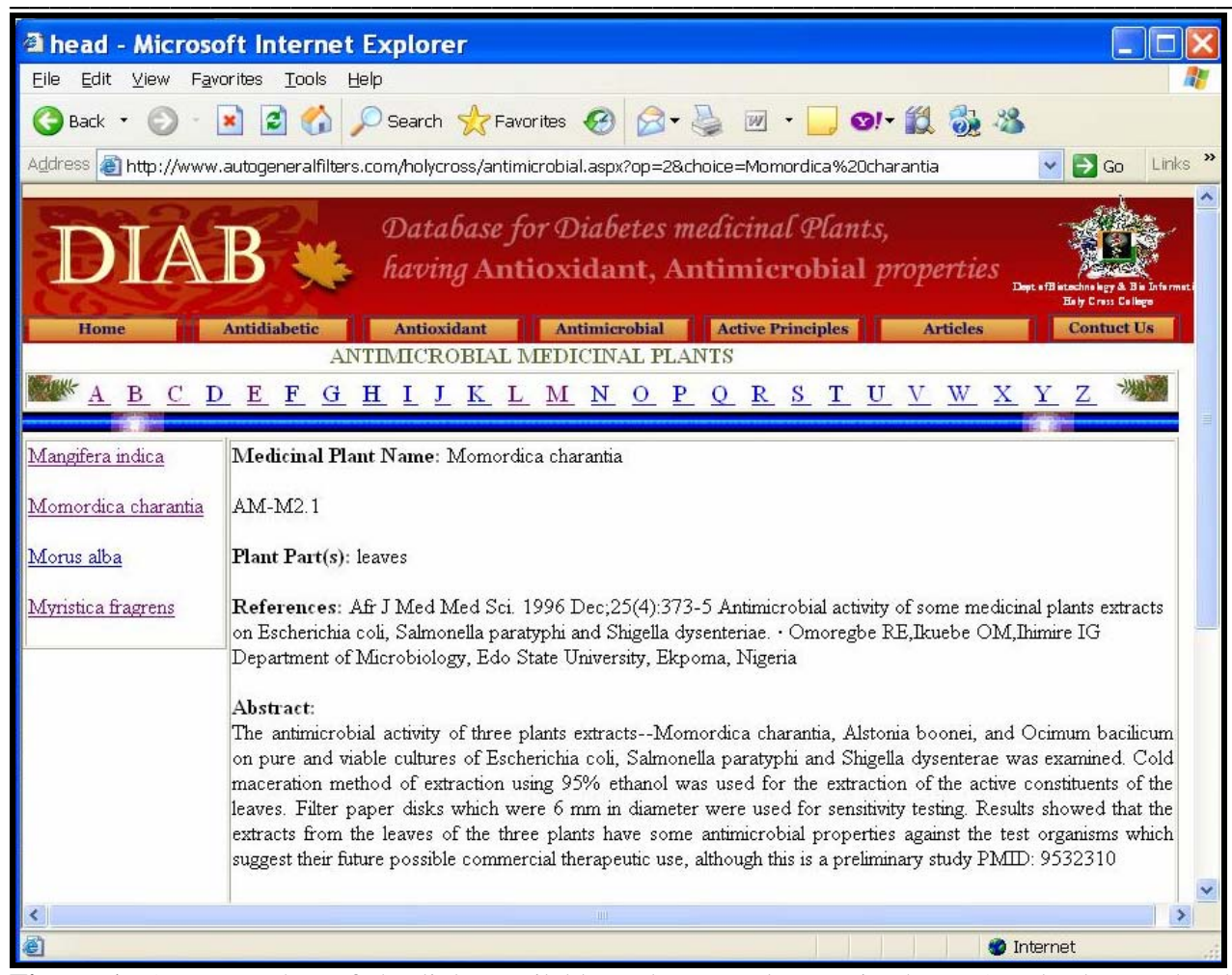

Figure 1: A screen shot of the links available and a record entry in the DIAB database. The three links (antidiabetic, antioxidant, antimicrobial) provide literature information. The active principles link can be accessed for information on compounds isolated. Full text articles can be downloaded from the 'Articles' link. The record entry for antimicrobial activity of Momordia charantia is shown.

\section{Future development:}

We plan to refine and keep updating this database and hopefully a link solely for indigenous plants will be made available in the nearest future. The database will also be modified to develop provisions to search the database to identify plants of interest using keywords.

\section{Acknowledgement:}

This project is funded by BTIS (Biotechnology information system), DBT (Department of Biotechnology), Ministry of Science and Technology, Government of India, India.

\section{References:}

[01] R. J. Marles, et al., Phytomedicine, 2:137(1995)
[02] C. L. Clark, Diabetes Care, 21:3 (1999)

[03] R. A. Arky, Complications of Diabetes mellitus, Philadelphia, Saunders WB., 16 (1982)

[04] M. K. Rai, Ancient Sci Life, 14:42 (1995)

[05] S. K. Mukherjee, J Diabed Assoc Ind., 21:97 (1995)

[06] http://www.ncbi.nlm.nih.gov/entrez/query.fcgi?d $\mathrm{b}=$ PubMed

[07] http://www.sciencedirect.com

[08] http://www.biomedcentral.com

[09] http://www.springerlink.com

[10] http://www.scirus.com

[11] http://www3.interscience.wiley.com/cgibin/home

Edited by P. Kangueane

License statement: This is an open-access article, which permits unrestricted use, distribution, and reproduction in any medium, for non-commercial purposes, provided the original author and source are credited. 